\title{
Surveillance de la rougeole au Canada : 2018
}

\author{
Cameron Coulby' ${ }^{1}$ Francesca Reyes Domingo ${ }^{1 *}$, Joanne Hiebert ${ }^{2}$, Diane MacDonald ${ }^{1}$
}

\section{Résumé}

Contexte : La rougeole a été éliminée au Canada en 1998. Chaque année, l'Agence de la santé publique du Canada compile des données épidémiologiques à l'intention de l'Organisation panaméricaine de la santé (OPS) pour vérifier que la rougeole est toujours éliminée au Canada. Cet article a pour objectif de présenter un résumé épidémiologique de l'activité associé à la rougeole déclarée au Canada en 2018 , et de fournir des données visant à faciliter la vérification continue du statut d'élimination de la rougeole au Canada.

Méthodes : Les données de surveillance de la rougeole ont été recueillies par le Système canadien de surveillance de la rougeole et de la rubéole (SCSRR). Une analyse descriptive des données démographiques et des facteurs de risque a été réalisée. Les caractéristiques des éclosions ont été résumées et une analyse des résultats de génotypage a été effectuée. Les données de surveillance de 2018 ont été évaluées par rapport aux critères essentiels de l'OPS pour vérifier le statut d'élimination de la rougeole.

Résultats : En 2018, 29 cas de rougeole ont été rapportés dans cinq provinces canadiennes, soit un taux d'incidence de 0,8 cas pour 1000000 personnes. Sur ces 29 cas, 16 ont été importés et cinq ont résulté en transmission subséquente au Canada. Le taux d'incidence ajusté selon l'âge était le plus élevé chez les personnes de moins d'un an (10,2 cas pour 1000000 personnes, $n=4$ ). Seuls neuf cas ont été considérés comme étant à jour pour la vaccination contre la rougeole, et 11 cas ont été hospitalisés. Les données de génotypage étaient disponibles pour la plupart des cas de rougeole $(n=27)$; il s'agissait de génotypes en circulation dans le monde en 2018. Le Canada a satisfait entièrement ou partiellement trois des quatre critères de l'OPS pour la vérification de l'élimination de la rougeole.

Conclusion : Bien que l'importation de la rougeole et les zones à faible couverture vaccinale constituent un défi pour le maintien du statut d'élimination de la rougeole au Canada, les données ne démontrent pas le rétablissement de la transmission endémique du virus de la rougeole. Le Canada maintient son statut d'élimination de la rougeole.
Cette oeuvre est mise à la disposition selon les termes de la licence internationale Creative Commons Attribution 4.0

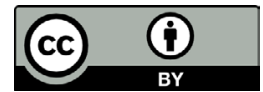

Affiliations

1 Centre de l'immunisation et des maladies respiratoires infectieuses, Agence de la santé publique du Canada, Ottawa, ON

${ }^{2}$ Laboratoire national de microbiologie, Agence de la santé publique du Canada, Winnipeg, MB

${ }^{\star}$ Correspondance : phac.vpd-mev.aspc@canada.ca

Citation proposée : Coulby C, Reyes Domingo F, Hiebert J, MacDonald D. Surveillance de la rougeole au Canada : 2018. Relevé des maladies transmissibles au Canada 2020;46(4):87-94.

https://doi.org/10.14745/ccdr.v46i04a04f

Mots-clés : rougeole, santé en voyage, surveillance, élimination de la rougeole, vaccination

\section{Introduction}

La rougeole est l'une des maladies infectieuses humaines les plus contagieuses. Elle était responsable d'environ 2,6 millions de décès chaque année avant l'introduction du vaccin contre la rougeole en 1963 (1). La rougeole a été éliminée au Canada en 1998 (2). L'Organisation mondiale de la santé (OMS) définit l'élimination comme l'absence de transmission endémique de la rougeole dans une zone géographique donnée, pendant une période supérieure ou égale à 12 mois, en présence d'un système de surveillance efficace qui permet le génotypage du virus identifié dans les cas confirmés de rougeole (3).
La rougeole reste néanmoins un important enjeu mondial de santé publique. En 2018, environ 9,8 millions de cas de rougeole et plus de 140000 décès liés à la rougeole ont été rapportés dans le monde (4). Ceci représente une hausse de plus de $40 \%$ du nombre de cas de rougeole et de près de $30 \%$ du nombre de décès liés à la rougeole au niveau mondial, par rapport à 2017 (5). La plupart des décès liés à la rougeole sont survenus chez les enfants âgés de moins de cinq ans (1).

Alors que le virus de la rougeole continue de circuler dans le monde, le risque de son importation au Canada subsiste. Le taux de couverture vaccinale pour la première dose du vaccin 
contenant le virus de la rougeole chez les enfants âgés de deux ans est estimé à $90 \%$, ce qui est inférieur au taux minimal de couverture vaccinale (95\%) nécessaire pour l'ensemble des cohortes démographiques permettant de prévenir la transmission soutenue de la rougeole $(2,6)$. La rougeole doit faire l'objet d'une surveillance nationale régulière et renforcée pour détecter rapidement les cas et les éclosions, et appliquer les mesures de santé publique nécessaires pour endiguer sa transmission. La surveillance renforcée s'exerce par l'entremise du Système canadien de surveillance de la rougeole et de la rubéole (SCSRR), lui-même coordonné par le Centre de l'immunisation et des maladies respiratoires infectieuses ainsi que le Laboratoire national de microbiologie (LNM) de l'Agence de la santé publique du Canada (ASPC). La surveillance repose sur la collecte hebdomadaire de données sur les cas confirmés de rougeole (7) auprès des 13 provinces et territoires (8).

Le Canada est engagé à éliminer la rougeole. Pour ce faire, des objectifs de couverture vaccinale et des cibles de réduction des maladies évitables par la vaccination, fondés sur les normes internationales et sur les pratiques exemplaires, ont été établis dans le cadre de la Stratégie nationale d'immunisation de 2016 à 2021 (9). Ces objectifs et cibles nationaux s'inscrivent dans les objectifs d'élimination des maladies de l'OMS et du Plan mondial d'action pour les vaccins, et reflètent le contexte canadien. En vertu de cette stratégie, le Canada vise une couverture vaccinale de $95 \%$ (et, de ce fait, l'élimination de la rougeole) avec l'administration d'une dose du vaccin contre la rougeole, les oreillons et la rubéole (ROR) avant l'âge de deux ans, et une couverture vaccinale de $95 \%$ avec l'administration de deux doses du vaccin ROR avant l'âge de sept ans. Les provinces et les territoires recommandent que la première dose du vaccin contenant le virus de la rougeole soit administrée à 12 mois et que la seconde dose soit administrée entre 18 mois et six ans (10). Les provinces et les territoires, à qui incombe la prestation des services de santé, notamment les programmes de vaccination, ont tous souscrit aux objectifs et cibles nationaux.

Ce rapport de surveillance a pour objectif de présenter un résumé épidémiologique de l'activité associée à la rougeole au Canada en 2018, et de fournir des données visant à faciliter la vérification continue du statut d'élimination de la rougeole au Canada.

\section{Méthodes}

\section{Données de surveillance}

Les cas confirmés de rougeole répondant à la définition nationale de cas (7) sont rapportés par les provinces et les territoires chaque semaine à l'ASPC par l'entremise du SCSRR. Tous les cas confirmés de rougeole dont l'éruption cutanée est apparue entre le $1^{\text {er }}$ janvier 2018 et le 31 décembre 2018 sont inclus dans ce rapport. La première semaine épidémiologique se termine le premier samedi de l'année. Le Centre de l'immunisation et des maladies respiratoires infectieuses attribue les semaines épidémiologiques en fonction de la date d'apparition des éruptions cutanées. Un processus de validation des données a été mené avec l'ensemble des provinces et des territoires. Celui-ci consistait à rechercher les données manquantes, à repérer les saisies incorrectes et à confirmer les valeurs auprès des provinces et territoires déclarants. Les cas pour lesquels il manquait des données ont été inclus dans l'analyse, au besoin. Les visiteurs du Canada chez qui la rougeole a été diagnostiquée pendant leur séjour ont également été inclus dans l'analyse.

\section{Génotypage}

Le génotypage du virus est systématiquement effectué par le LNM pour tous les cas confirmés au Canada pour lesquels des échantillons viraux sont disponibles. Le génotypage consiste à séquencer certaines cibles du génome de la rougeole, conformément aux lignes directrices de l'OMS $(11,12)$. Les séquences virales de la rougeole sont consignées dans la base de données Measles Nucleotide Surveillance (MeaNS) de l'OMS, puis comparées à certaines souches nommées et à des séquences documentées par d'autres membres du Réseau mondial de laboratoires sur la rougeole $(12,13)$.

\section{Analyse}

Des analyses épidémiologiques descriptives ont été réalisées à partir des champs existants de la base de données du SCSRR (7). L'analyse par sous-groupes n'a pas été effectuée en raison du faible nombre de cas rapportés en 2018.

Les cas âgés d'un à six ans qui ont reçu au moins une dose (documentée) du vaccin contenant le virus de la rougeole étaient considérés comme ayant un statut vaccinal à jour. Les cas âgés de sept ans ou plus et ceux nés après 1970 étaient considérés à jour pour la vaccination contre la rougeole s'ils avaient reçu deux doses (documentées) du vaccin contre la rougeole, et sous-vaccinés s'ils avaient reçu une seule dose (documentée) du vaccin $(9,14)$. On présume qu'un adulte né avant 1970 a acquis naturellement l'immunité contre la rougeole et est ainsi considéré à jour pour la vaccination contre la rougeole. Un enfant de moins d'un an était considéré à jour, sans égard à son statut vaccinal, à moins qu'il ait voyagé à l'extérieur de l'Amérique du Nord. Dans ce cas, le vaccin peut lui être administré après l'âge de six mois (15).

Un cas était considéré comme hospitalisé seulement s'il avait été admis à l'hôpital à cause de la rougeole ou à cause de complications liées à la maladie. Si le cas avait seulement été admis au service des urgences, il n'était pas considéré comme hospitalisé.

La source d'exposition a été déterminée par la province ou le territoire déclarant au cours de l'enquête de santé publique. Les sources d'exposition ont été catégorisées comme suit : extérieure du Canada (importée); au Canada et liée à un cas importé (liée à l'importation); au Canada et liée à un cas d'origine inconnue; ou source inconnue/sporadique. 
Les éclosions de rougeole, définies comme l'apparition de deux cas confirmés ou plus liés sur le plan épidémiologique ou virologique, ou les deux, ont été décrites à partir des informations disponibles (14). Les taux d'incidence ont été calculés à l'aide des estimations de la population au $1^{\text {er }}$ juillet 2018 de Statistique Canada.

\section{Résultats}

Au total, 29 cas confirmés de rougeole (soit un taux d'incidence de 0,8 cas pour 1000000 personnes), répartis sur cinq provinces, ont été rapportés au Canada en 2018 . Sur ces 29 cas confirmés, 27 ont été génotypés. Les génotypes identifiés étaient B3 ( $n=7)$ et D8 ( $n=20)$, deux génotypes circulant dans le monde en 2018 (4). La figure 1 illustre la répartition des cas de rougeole par semaine épidémiologique d'apparition de l'éruption cutanée, selon l'ordre chronologique des éclosions, selon le génotype et selon la province ou le territoire déclarant. Au total, 28 cas ont été confirmés en laboratoire et un cas avait un lien épidémiologique avec un cas confirmé en laboratoire.

Figure 1 : Nombre de cas de rougeole rapportés $(N=29)$ par semaine épidémiologique d'apparition de l'éruption cutanée, selon l'ordre chronologique des éclosions, selon le génotype et selon la province ou le territoire déclarant, au Canada, en 2018

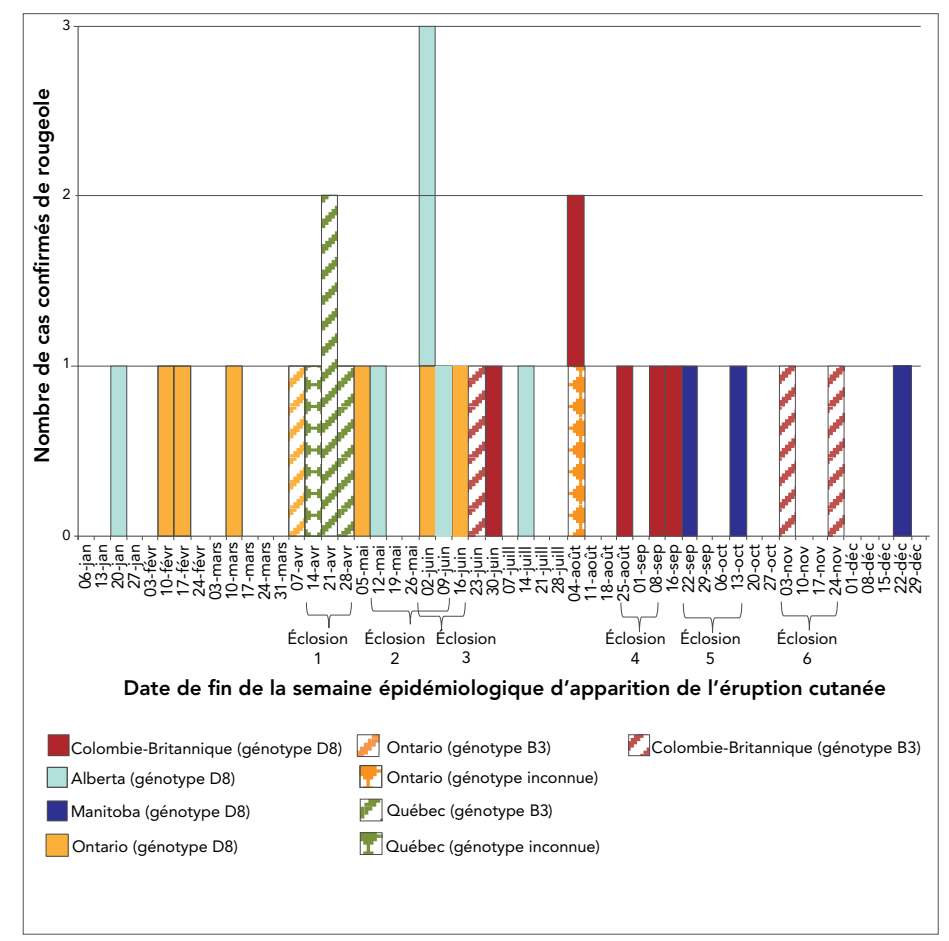

Les données sur l'âge, le sexe et la province ou le territoire de résidence étaient disponibles pour tous les cas de rougeole rapportés en 2018. L'âge des cas variait de moins d'un an à 53 ans, et l'âge médian était de 21 ans. Le groupe d'âge le plus touché était celui des 25 à 44 ans $(n=11)$, suivi de celui des 15 à 24 ans $(n=8)$. Le taux d'incidence le plus élevé était observé chez les moins d'un an, avec 10,2 cas pour 1000000 personnes (figure 2). Trois quarts des cas $(n=22)$ étaient des femmes.

Figure 2 : Cas confirmés de rougeole $(N=29)$ et taux d'incidence (pour 1000000 personnes) par groupe d'âge, au Canada, en 2018

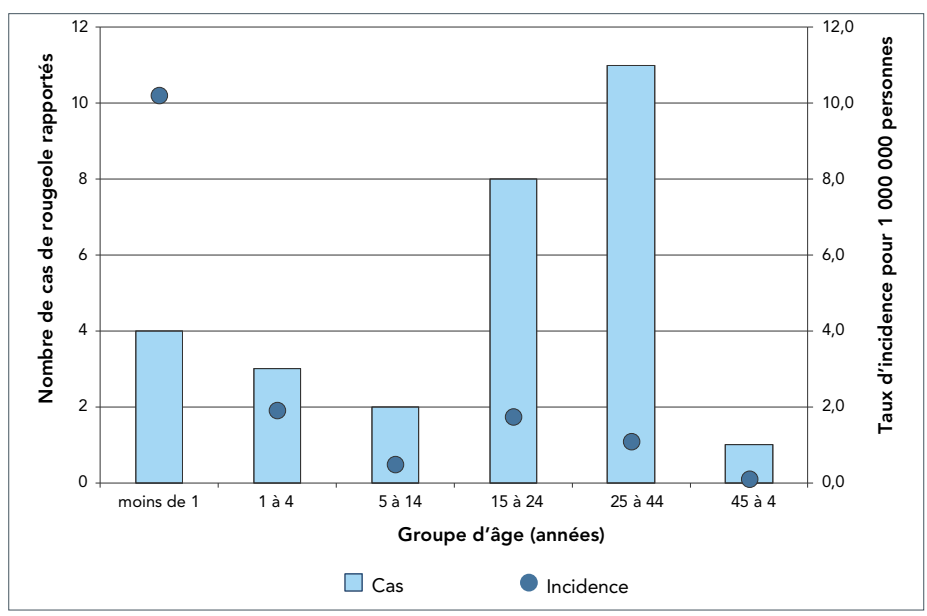

Cinq provinces canadiennes ont rapporté des cas de rougeole en 2018 : I'Ontario ( $n=9$, dont un visiteur de l'Ontario), la Colombie-Britannique $(n=8)$, l'Alberta $(n=6)$, le Québec $(n=4)$ et le Manitoba $(n=2)$. Dans ces provinces, les taux $d$ 'incidence variaient de 0,5 à 1,6 cas pour 1000000 personnes.

\section{Vaccination}

Pour deux tiers $(n=20)$ des cas, le statut vaccinal n'était pas à jour selon leur âge. Parmi ceux-ci la documentation sur le statut vaccinal confirmait ce statut pour 12 cas. Trois sur les 20 cas étaient âgés entre six mois et un an et avaient voyagé à l'extérieur de l'Amérique du Nord sans avoir reçu la première dose du vaccin contre la rougeole, tel que préconisé par les recommandations vaccinales actuelles $(15,16)$. Les antécédents de vaccination étaient inconnus ou manquants pour huit cas; ces derniers cas étaient nés après 1970, soit l'année utilisée pour déterminer l'immunité naturelle présumée pour la rougeole (15).

Sur les 29 cas de rougeole déclarés en 2018, neuf avaient un statut vaccinal à jour selon leur âge, et huit ont déclaré disposer de documents attestant être à jour selon leur âge (notamment un nourrisson de moins d'un an qui n'était pas vacciné et qui n'avait pas voyagé à l'extérieur de l'Amérique du Nord) (figure 3). Un cas dont les antécédents de vaccination étaient inconnus était né avant 1970 (et, par conséquent, considéré comme ayant un statut vaccinal à jour) et n'avait pas voyagé en dehors de l'Amérique du Nord (15).

\section{Hospitalisation}

Les données sur les hospitalisations étaient disponibles pour 24 des 29 cas rapportés en 2018. Au total, 11 cas ont été hospitalisés, ce qui représente un taux d'hospitalisation de 0,3 cas pour 1000000 personnes. L'âge médian des cas hospitalisés était 15 ans (intervalle de 4 à 51 ans). Parmi les 
cas hospitalisés, les informations sur la vaccination étaient disponibles pour huit cas; six n'avaient pas reçu de dose (documentée) du vaccin contre la rougeole, mais deux étaient considérés complètement vaccinés.

Figure 3 : Statut vaccinal des cas confirmés de rougeole $(\mathrm{N}=29)$ au Canada, en 2018

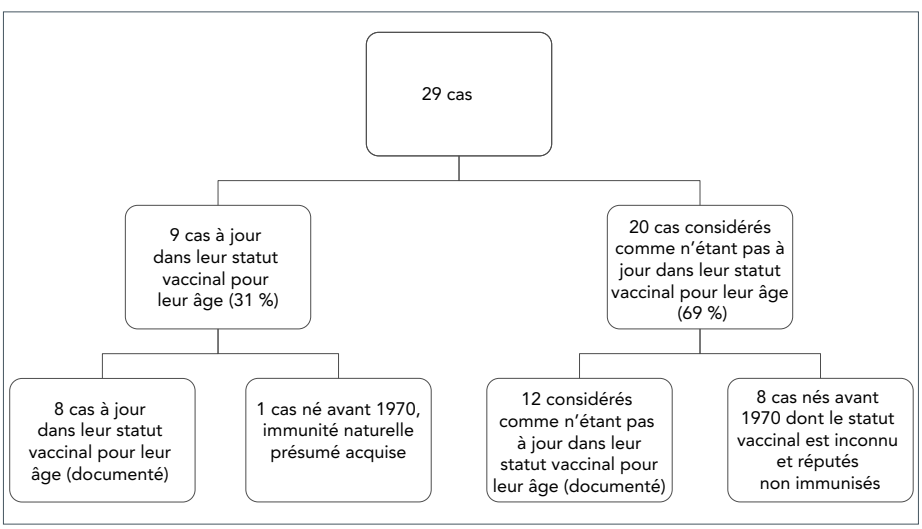

\section{Sources d'exposition}

Seize cas ont été importés au Canada en 2018 suite à une exposition lors d'un voyage dans les pays ou les régions suivants : Inde $(n=7)$, Ukraine $(n=4)$, Brésil $(n=1)$, Philippines $(n=1)$, Roumanie $(n=1)$, Asie du Sud-Est (sauf Inde, $n=1)$ et
Ouganda $(n=1)$. Quatorze des cas importés ont fait l'objet d'un génotypage et les génotypes détectés correspondaient à ceux endémiques dans les pays où les cas avaient été exposés, ou étaient compatibles avec ceux de la base de données MeaNS de l'OMS pour ces pays (13).

Sur les 16 cas importés, cinq ont résulté en transmission subséquente au Canada, occasionnant l'apparition de sept cas. Les cas importés et liés à une importation représentaient le trois quarts $(n=23)$ des cas en 2018. Parmi les cas qui n'étaient ni importés ni liés à l'importation $(n=6)$, quatre n'avaient pas d'antécédents récents de voyage ou de liens connus avec d'autres cas confirmés de rougeole; un cas était exposé à un autre cas d'origine inconnue au Canada; un second cas ne pouvait être classé comme importé ou acquis au Canada, car la période d'exposition a eu lieu à la fois au Canada et dans un autre pays où des cas de rougeole ont été rapportés. Les six cas ont été génotypés; les génotypes détectés étaient $D 8(n=5)$ et B3 $(n=1)$, deux génotypes circulants à travers le monde.

\section{Éclosions}

Six éclosions de rougeole ont été rapportées au Canada en 2018 (voir tableau 1 pour plus de détails). Il s'agissait de petites éclosions (deux à quatre cas par éclosion) et la transmission était limitée aux contacts familiaux ou aux contacts étroits du cas index. Aucune éclosion n'a présenté une transmission au-delà de

\section{Tableau 1 : Résumé des éclosions de rougeole au Canada $(\mathrm{N}=6)$ selon la date d'apparition de l'éruption cutanée, en 2018}

\begin{tabular}{|c|c|c|c|c|c|}
\hline $\begin{array}{c}N^{\circ} \\
\text { d'éclosion }\end{array}$ & $\begin{array}{l}\text { Province } \\
\text { ou } \\
\text { territoire }\end{array}$ & $\begin{array}{c}\text { Nombre } \\
\text { de case }\end{array}$ & $\begin{array}{c}\text { Date de fin } \\
\text { de la semaine } \\
\text { épidémiologique } \\
\text { d'apparition de } \\
\text { l'éruption cutanée } \\
\text { du cas index }\end{array}$ & $\begin{array}{l}\text { Génotype } \\
\text { (souche) }\end{array}$ & Description \\
\hline 1 & Québec & 4 & 15 avril & $\begin{array}{l}\text { B3 (MVs/ } \\
\text { Dublin. } \\
\text { IRL/8.16/) }\end{array}$ & $\begin{array}{l}\text { Le cas index a rapporté avoir voyagé en Roumanie pendant la période } \\
\text { d'exposition. Trois cas secondaires parmi les contacts familiaux du cas index ont } \\
\text { été rapportés par la suite. Aucun des cas n'était vacciné. }\end{array}$ \\
\hline 2 & Alberta & 2 & 13 mai & D8 & $\begin{array}{l}\text { Le cas index a rapporté avoir voyagé en Inde pendant la période d'exposition. } \\
\text { Un cas secondaire, contact étroit du cas index, a été rapporté par la suite. Les } \\
\text { antécédents de vaccination du cas index étaient inconnus. Le cas secondaire } \\
\text { n'était pas vacciné. }\end{array}$ \\
\hline 3 & Ontario & 2 & 3 juin & $\begin{array}{l}\text { D8 (MVs/ } \\
\text { Gir Somnath. } \\
\text { IND/42.16/) }\end{array}$ & $\begin{array}{l}\text { Le cas index a rapporté avoir voyagé en Ukraine pendant la période } \\
\text { d'exposition. Un cas secondaire, contact étroit du cas index, a été rapporté par } \\
\text { la suite. Les antécédents de vaccination du cas index étaient inconnus. Le cas } \\
\text { secondaire n'était pas vacciné. }\end{array}$ \\
\hline 4 & $\begin{array}{l}\text { Colombie- } \\
\text { Britannique }\end{array}$ & 2 & 26 août & $\begin{array}{l}\text { D8 (MVs/ } \\
\text { Osaka. } \\
\text { JPN/29.15/) }\end{array}$ & $\begin{array}{l}\text { Le cas index a rapporté ne pas avoir voyagé en dehors du Canada pendant la } \\
\text { période d'exposition, mais a pu être exposé à un cas de rougeole international } \\
\text { dans un port canadien. Un cas secondaire, contact du cas index, a été rapporté } \\
\text { par la suite. Les deux cas étaient complètement vaccinés. }\end{array}$ \\
\hline 5 & Manitoba & 2 & 9 septembre & $\begin{array}{l}\text { D8 (MVs/ } \\
\text { Samut Sakhon. } \\
\text { THA/49.16) }\end{array}$ & $\begin{array}{l}\text { Le cas index a rapporté avoir voyagé en Asie du Sud-Est pendant la période } \\
\text { d'exposition. Un cas secondaire, contact étroit du cas index, a été rapporté par } \\
\text { la suite. Le cas index n'était pas vacciné. Le cas secondaire était complètement } \\
\text { vacciné. }\end{array}$ \\
\hline 6 & $\begin{array}{l}\text { Colombie- } \\
\text { Britannique }\end{array}$ & 2 & 4 novembre & B3 & $\begin{array}{l}\text { Le cas index a rapporté avoir voyagé aux Philippines. Un cas secondaire, } \\
\text { contact du cas index, a été rapporté par la suite. Les antécédents de } \\
\text { vaccination du cas index étaient inconnus. Le cas secondaire était } \\
\text { complètement vacciné. }\end{array}$ \\
\hline
\end{tabular}

Souche nommée par l'Organisation mondiale de la santé (OMS) est indiquée entre parenthèses, le cas échéant. Le système de nomination des souches par l'OMS constitue un système visant à

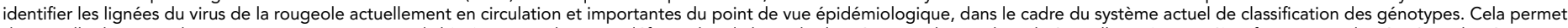

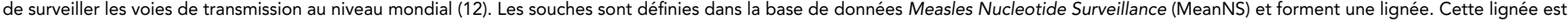
formée d'une souche virale bien définie. Cette dernière a été détectée à de multiples reprises en l'espace de deux ans, dans plusieurs pays. Les numéros d'accès à la base GenBank associés aux souches nommées sont KY013331, KY120864, LC072667 et MK079566. 
la deuxième génération. L'intervalle médian entre l'apparition de l'éruption cutanée du cas index et l'apparition des cas secondaires était de 13 jours (intervalle de neuf à 27 jours) pour toutes les éclosions. La période d'incubation pour un des cas était en dehors de l'intervalle escompté de sept à 21 jours; ce cas avait reçu des immunoglobulines peu de temps après l'exposition. Ceci aurait pu retarder l'apparition de la maladie sans la prévenir. Sur les 29 cas déclarés en 2018, 14 étaient liés à une éclosion.

\section{Discussion}

\section{Épidémiologie de la rougeole au Canada en 2018}

En 2018, 29 cas confirmés de rougeole ont été rapportés au Canada, ce qui est inférieur au nombre médian de cas déclarés depuis le début de la surveillance renforcée en 1998 (valeur médiane de 35 cas par an). Cette réduction du nombre de cas contrastait avec la tendance à la hausse des taux de mortalité imputables à la rougeole dans le monde. Aux États-Unis, le nombre de cas et d'éclosions de rougeole recensés en 2018 a augmenté par rapport à $2017(5,17)$. Sur les 16 cas de rougeole importés au Canada en 2018, seuls cinq ont donné lieu à une transmission secondaire, mettant ainsi en évidence l'absence de transmission soutenue.

Les cas importés et liés à l'importation constituaient la grande majorité des cas de rougeole en 2018. Ceci souligne le rôle que les voyages à l'étranger jouent dans la propagation de la rougeole au Canada. En effet la détection des deux principaux génotypes associés aux cas recensés au Canada, soit B3 et D8, étaient en circulation dans le monde en 2018. Ces génotypes circulaient également au niveau mondial en 2017 et ont été détectés au Canada (18). Cependant, les génotypes identifiés contiennent des séquences génétiquement liées et contiennent plusieurs souches. En ce qui a trait à l'épidémiologie moléculaire de la rougeole, les souches sont informatives. Les souches détectées en 2017, à l'exception de la souche nommée MVs/ Dublin.IRL/8.16 du génotype B3, n'ont pas été détectées en 2018.

Plus de deux tiers des cas de rougeole déclarés en 2018 n'étaient pas à jour dans leur statut vaccinal pour leur âge. Ceci souligne l'importance de suivre les recommandations vaccinales. Il est possible que la réaction immunitaire des sept cas dont le statut vaccinal était à jour, n'était pas adaptée. Il est également possible que l'immunité conférée par le vaccin contre la rougeole ait diminué à un tel point qu'elle ne protégeait plus contre la maladie, ou alors que le vaccin ait été stocké, manipulé ou administré de manière incorrecte $(19,20)$. Sur ces sept cas, deux avaient reçu des doses (documentées) du vaccin contre la rougeole dans un autre pays où les lignes directrices relatives au stockage et à l'administration du vaccin peuvent être différentes.
Plus d'un tiers (11 sur 29) des cas ont dû être hospitalisés, ce qui est conforme aux données publiées dans la littérature stipulant que l'infection à la rougeole peut mener à forme sévère de la maladie (1).

\section{Vérification de l'élimination de la rougeole par l'intermédiaire d'objectifs et de cibles nationaux et internationaux}

L'Organisation panaméricaine de la santé (OPS) a fixé quatre critères pour la vérification continue de l'élimination de la rougeole (21). Le Canada a satisfait entièrement ou partiellement à trois des quatre critères en 2018 (tableau 2). Les critères de l'OPS reposent sur l'enquête des cas suspects chez qui les symptômes sont compatibles à la rougeole. Cependant, au Canada, seuls les cas confirmés font l'objet d'une déclaration obligatoire. Ainsi, les données contenues dans cet article ne répondent qu'indirectement aux critères de l'OPS. Au Canada, le système national de surveillance de la rougeole est efficace puisqu'il permet de détecter les cas importés et liés à l'importation, ainsi que les cas dont la source d'exposition est inconnue. De plus, le statut d'élimination conféré par les critères internationaux permet d'affirmer que le Canada satisfait aux objectifs et cibles nationaux qu'il s'est fixés.

\section{Tableau 2 : Critères essentiels de l'Organisation panaméricaine de la santé pour la vérification de l'élimination de la rougeole}

\begin{tabular}{|c|c|c|}
\hline Critère & Indicateur & Description \\
\hline $\begin{array}{l}\text { Vérifier l'interruption } \\
\text { de la transmission de } \\
\text { cas endémiques de } \\
\text { rougeole pendant une } \\
\text { période d'au moins } \\
3 \text { ans à partir de la } \\
\text { date de survenue } \\
\text { du dernier cas } \\
\text { endémique connu, } \\
\text { alors qu'un système } \\
\text { de surveillance de } \\
\text { haute qualité est en } \\
\text { place. }\end{array}$ & $\begin{array}{l}\text { Aucun cas de } \\
\text { transmission } \\
\text { endémique. }\end{array}$ & $\begin{array}{l}\text { Critère satisfait. } \\
\text { Le Canada a obtenu le } \\
\text { statut d'élimination de la } \\
\text { rougeole en 1998. Depuis, } \\
\text { les données moléculaires } \\
\text { et épidémiologiques } \\
\text { continuent de démontrer } \\
\text { qu'aucune souche virale ne } \\
\text { s'est établie pendant une } \\
\text { période d'un an }(3,18,21,22) \text {. }\end{array}$ \\
\hline $\begin{array}{l}\text { Maintenir un système } \\
\text { de surveillance } \\
\text { de qualité élevée } \\
\text { suffisamment sensible } \\
\text { pour détecter les } \\
\text { cas importés et liés à } \\
\text { l'importation. }\end{array}$ & $\begin{array}{l}\text { Plus de deux } \\
\text { cas suspects } \\
\text { par tranche } \\
\text { de } 100000 \\
\text { personnes } \\
\text { ayant fait } \\
\text { l'objet d'une } \\
\text { enquête } \\
\text { adéquate. }\end{array}$ & $\begin{array}{l}\text { Critère partiellement } \\
\text { satisfait. } \\
\text { Le SCSRR permet d'identifier } \\
\text { les cas importés et liés à } \\
\text { l'importation qui répondent } \\
\text { à la définition de cas, mais } \\
\text { pas les cas suspects. }\end{array}$ \\
\hline $\begin{array}{l}\text { Vérifier l'absence } \\
\text { de souches de virus } \\
\text { endémiques de } \\
\text { la rougeole grâce } \\
\text { à la surveillance } \\
\text { virologique. }\end{array}$ & $\begin{array}{l}\text { Génotypage } \\
\text { du virus de } \\
\text { la rougeole } \\
\text { dans } 80 \% \text { des } \\
\text { éclosions. }\end{array}$ & $\begin{array}{l}\text { Critère satisfait. } \\
\text { Des données de génotypage } \\
\text { étaient disponibles pour } \\
\text { l'intégralité des éclosions } \\
\text { déclarées en } 2018 \text { (6). Des } \\
\text { données de génotypage } \\
\text { étaient également } \\
\text { disponibles pour l'intégralité } \\
\text { des cas sporadiques de } \\
\text { rougeole (sans lien avec une } \\
\text { éclosion). }\end{array}$ \\
\hline
\end{tabular}


Tableau 2 : Critères essentiels de l'Organisation panaméricaine de la santé pour la vérification de l'élimination de la rougeole (suite)

\begin{tabular}{|c|c|c|}
\hline Criterion & Indicator & Description \\
\hline $\begin{array}{l}\text { Vérifier que la } \\
\text { population est } \\
\text { adéquatement } \\
\text { immunisée. }\end{array}$ & $\begin{array}{l}95 \% \text { des } \\
\text { cohortes } \\
\text { âgées de } 1 \\
\text { à } 40 \text { ans ont } \\
\text { reçu un vaccin } \\
\text { contre la } \\
\text { rougeole. }\end{array}$ & $\begin{array}{l}\text { Critère non satisfait. } \\
\text { Le Canada mesure } \\
\text { actuellement (aux deux } \\
\text { ans) le taux de couverture } \\
\text { vaccinale de la rougeole } \\
\text { pour les enfants âgés } \\
\text { de deux et sept ans. Par } \\
\text { conséquent le Canada n'est } \\
\text { pas en mesure d'évaluer la } \\
\text { couverture vaccinale de la } \\
\text { rougeole chez les personnes } \\
\text { âgées de } 1 \text { à } 40 \text { ans. D'après } \\
\text { l'Enquête nationale sur } \\
\text { la couverture vaccinale } \\
\text { des enfants de } 2017, \text { la } \\
\text { couverture vaccinale pour } \\
\text { la première dose du vaccin } \\
\text { contenant la rougeole chez } \\
\text { les enfants âgés de deux ans } \\
\text { était estimée à } 90 \% \text { (6). }\end{array}$ \\
\hline
\end{tabular}

Abréviation : SCSRR, Système canadien de surveillance de la rougeole et de la rubéole

Le Canada ne satisfait pas au critère relatif à la couverture vaccinale de la rougeole. Le Canada mesure actuellement (aux deux ans) le taux de couverture vaccinale de la rougeole pour les enfants âgés de deux et sept ans. Par conséquent le Canada n'est pas en mesure d'évaluer la couverture vaccinale de la rougeole chez les personnes âgées de 1 à 40 ans tel qu'énoncé dans le cadre d'élimination de l'OPS. En 2017, le taux de couverture vaccinale de la rougeole chez les enfants âgés de deux ans était estimé à $90 \%$, ce qui est inférieur à I'indicateur établi par l'OPS (95\%) (6). Cette estimation provient d'une enquête ayant collecté des données issues des carnets de vaccination conservés par les parents, et dans lesquels certains renseignements peuvent être incomplets, erronés ou tout simplement manquants. II est fort probable que l'enquête sousestime la couverture car les doses de vaccins administrées sans date ou avec une date invalide ne sont pas comptabilisés dans le calcul de la couverture vaccinale.

\section{Limites}

Seuls les cas de rougeole ayant été en contact avec le système de santé canadien sont inclus dans le cadre de la surveillance renforcée de la rougeole. De ce fait, il est possible que les visiteurs au Canada qui n'ont pas recours aux soins médicaux ne soient pas capturés. Cependant, la plupart des chaînes de transmission étaient le résultat de cas importés connus en 2018.

Le SCSRR ne collecte pas d'informations sur la mortalité ni sur la morbidité (p.ex. durée d'hospitalisation, séquelles), ce qui ne permet pas d'avoir un portrait global du fardeau de la rougeole.

\section{Conclusion}

Tant au Canada qu'à l'étranger, le maintien d'un taux élevé de couverture vaccinale de la rougeole nécessite un effort soutenu de santé publique et constitue un élément essentiel pour obtenir et conserver le statut d'élimination. Bien que l'importation de la rougeole et les zones à faible couverture vaccinale constituent un défi pour le maintien du statut d'élimination de la rougeole au Canada, les données ne démontrent pas le rétablissement de la transmission endémique du virus de la rougeole. Ce constat est corroboré par le fait que, dans l'ensemble, le taux de couverture vaccinale est élevé. De plus, le nombre de cas secondaires induits par les cas importés est faible, et les données de laboratoire disponibles indiquent que les cas résultent de souches virales circulant globalement en 2018.

\section{Déclaration des auteurs}

C. C. - Méthodologie, logiciel, analyse formelle, enquête, organisation des données, rédaction de la version initiale, rédaction de la version définitive, révision, édition, visualisation F. R. D. - Conceptualisation, méthodologie, analyse formelle, rédaction de la version initiale, rédaction de la version définitive, révision, édition, administration de projet

J. H. - Méthodologie, validation, enquête, organisation des données, rédaction de la version initiale, rédaction de la version définitive, édition

D. M. - Conceptualisation, méthodologie, rédaction, révision, édition, administration de projet

\section{Conflit d'intérêts}

Aucun.

\section{Remerciements}

Les auteurs tiennent à remercier chaleureusement leurs partenaires provinciaux et territoriaux de surveillance et d'analyse en laboratoire pour leur collaboration et leurs efforts continus visant à fournir et valider les données recueillies par le Système canadien de surveillance de la rougeole et de la rubéole (SCSRR), à acheminer les échantillons aux fins de surveillance moléculaire (génotypage) et réviser le contenu de ce rapport.

\section{Financement}

Ces travaux ont été soutenus par l'Agence de la santé publique du Canada. 


\section{Références}

1. Organisation mondiale de la Santé (OMS). Rougeole Genève (CH) : OMS. http://www.who.int/news-room/fact-sheets/ detail/measles

2. King A, Varughese P, De Serres G, Tipples GA, Waters J; Working Group on Measles Elimination. Measles elimination in Canada. J Infect Dis 2004 May;189 Suppl 1:S236-42. DOI PubMed

3. L'élimination de la rougeole, de la rubéole et du syndrome de rubéole congénitale au Canada: Sommaire. Ottawa (ON): Gouvernement du Canada. https://www. canada.ca/en/public-health/services/immunization/ vaccine-preventable-diseases/elimination-measle s-rubella-congenital-rubella-syndrome-canada-documentat ion-verification-report.html L'élimination de la rougeole, de la rubéole et du syndrome de ru-béole congénitale au Canada

4. Patel MK, Dumolard L, Nedelec Y, Sodha SV, Steulet C, Gacic-Dobo M, Kret-singer K, McFarland J, Rota PA, Goodson JL. Progress toward regional measles eliminationworldwide, 2000-2018. MMWR Morb Mortal Wkly Rep 2019 Dec;68(48):1105-11. DOI PubMed

5. Dabbagh A, Laws RL, Steulet C, Dumolard L, Mulders MN, Kretsinger K, Alexander JP, Rota PA, Goodson JL. Progress toward regional measles elimination- worldwide, 20002017. MMWR Morb Mortal Wkly Rep 2018 Nov;67(47):13239. DOI PubMed

6. Faits saillants de l'Enquête nationale sur la couverture vaccinale des enfants (ENCVE), 2017. Ottawa (ON) : Gouvernement du Canada. https://www.canada.ca/ $\mathrm{fr} / \mathrm{services/sante/publications/vaccins-immunisation/}$ couverture-vaccinale-enfants-canadiens-faits-saillants-eng uete-nationale-couverture-vaccinale-enfants-2017.html

7. Agence de la santé publique du Canada. Définitions nosologiques des maladies transmissibles faisant l'objet d'une surveillance nationale. Relevé des maladies transmissibles au Canada 2009;35-Suppl 2:76-9. https://www. canada.ca/content/dam/phac-aspc/migration/phac-aspc/ publicat/ccdr-rmtc/09pdf/35s2-fra.pdf

8. Système canadien de surveillance de la rougeole et de la rubéole. Ottawa (ON): Gouvernement du Canada. https:// www.canada.ca/fr/sante-publique/services/surveillance. html\#a19

9. Objectifs nationaux de couverture vaccinale et cibles nationales de réduction des maladies évitables par la vaccination d'ici 2025 : Cibles de réduction des maladies évitables par la vaccination d'ici 2025 : Maladies en voie d'élimination : maintenir l'élimination. Ottawa (ON) : Gouvernement du Canada. https://www.canada.ca/fr/ sante-publique/services/priorites-immunization-et-vaccins/ strategie-nationale-immunisation/vaccination-objectif s-nationaux-couverture-vaccinale-cibles-nationales-reduction -maladies-evitables-2025.html
10. Programmes de vaccination systématique et de rattrapage des provinces et des territoires pour les nourrissons et les enfants au Canada. Ottawa (ON) : Gouvernement du Canada; 2018. https://www.canada.ca/fr/sante-publique/ services/renseignements-immunisation-provinces-etterritoires/programmes-vaccination-systematique-provin ces-territoires-nourrissons-enfants.html

11. Organisation mondiale de la Santé (OMS). Programme élargi de vaccination (PEV). Nomenclature relative à la description des caractéristiques génétiques des virus rougeoleux sauvages: standardisation. Wkly Epidemiol Rec 1998 Aug;73(35):265-9. https://apps.who.int/iris/ bitstream/handle/10665/230557/WER7335_265-269. PDF? sequence $=1$ \&isAllowed $=y$

12. Organisation mondiale de la Santé (OMS). La diversité génétique des virus rougeoleux de type sauvage et la base de données MeaNS (Measles Nucleotide Surveillance). Wkly Epidemiol Rec 2015 Jul;90(30):373-80. https://apps.who. int/iris/bitstream/handle/10665/242393/WER9030_373-380. PDF? sequence $=1$ \&isAllowed $=y$

13. Rota PA, Brown K, Mankertz A, Santibanez S, Shulga S, Muller CP, Hübschen JM, Siqueira M, Beirnes J, Ahmed H, Triki H, Al-Busaidy S, Dosseh A, Byabamazima C, Smit S, Akoua-Koffi C, Bwogi J, Bukenya H, Wairagkar N, Ramamurty N, Incomserb P, Pattamadilok S, Jee Y, Lim W, Xu W, Komase K, Takeda M, Tran T, Castillo-Solorzano C, Chenoweth P, Brown D, Mulders MN, Bellini WJ, Featherstone D. Global distribution of measles genotypes and measles molecular epidemiology. J Infect Dis 2011 Jul;204 Suppl 1:S514-23. DOl PubMed

14. Groupe de travail pour l'élimination de la rougeole et de la rubéole (GTERR), Santé Canada, Agence santé publique du Canada. Lignes directrices pour la prévention et le contrôle des éclosions de rougeole au canada: Une déclaration d'un comité consultatif (DCC) Groupe de travail pour l'élimination de la rougeole et de la rubéole (GTERR). Relevé des maladies transmissibles au Canada;39(ACS-3):1-58. DOI

15. Vaccin contre la rougeole: Guide canadien d'immunisation : Tableau 1: Critères d'immunité contre la rougeole. Ottawa (ON) : Gouvernement du Canada; 2018. https://www. canada.ca/fr/sante-publique/services/publications/vie-saine/ guide-canadien-immunisation-partie-4-agents-immunisationactive/page-12-vaccin-rougeole.html

16. Immunisation des voyageurs: Guide canadien d'immunisation. Ottawa (ON) : Gouvernement du Canada; 2017. https://www.canada.ca/en/ public-health/services/publications/healthy-living/ canadian-immunization-guide-part-3-vaccination-specificpopulations/page-9-immunization-travellers.html

17. The United States Centre for Disease Control and Prevention. Measles (Rubeola): measles cases and outbreaks. Atlanta (GA): U.S. Department of Health \& Human Services; 2019. https://www.colc.gov/measles/cases-outbreaks.html 
18. Santé Canada. Surveillance de la rougeole au Canada : 2017. Ottawa (ON) : Gouvernement du Canada; 2018. https:// www.canada.ca/fr/sante-publique/services/publications/ maladies-et-affections/surveillance-de-la-rougeolecanada-2017.html

19. Breakwell L, Moturi E, Helgenberger L, Gopalani SV, Hales C, Lam E, Sharapov U, Larzelere M, Johnson E, Masao C, Setik E, Barrow L, Dolan S, Chen TH, Patel M, Rota P, Hickman C, Bellini W, Seward J, Wallace G, Papania M. Measles outbreak associated with vaccine failure in adults - Federated States of Micronesia, February-August 2014. MMWR Morb Mortal Wkly Rep 2015 Oct;64(38):1088-92. DOI PubMed
20. Hinman AR, Orenstein WA, Mortimer EA Jr. When, where, and how do immunizations fail? Ann Epidemiol 1992 Nov;2(6):805-12. DOl PubMed

21. Pan-American Health Organization. Plan of action. Documentation and verification of measles, rubella and congenital rubella syndrome elimination in the region of the Americas. Washington (DC): PAHO; 2010.6

\section{PRÉVENIR LA PROPAGATION DE LA COVID-19}

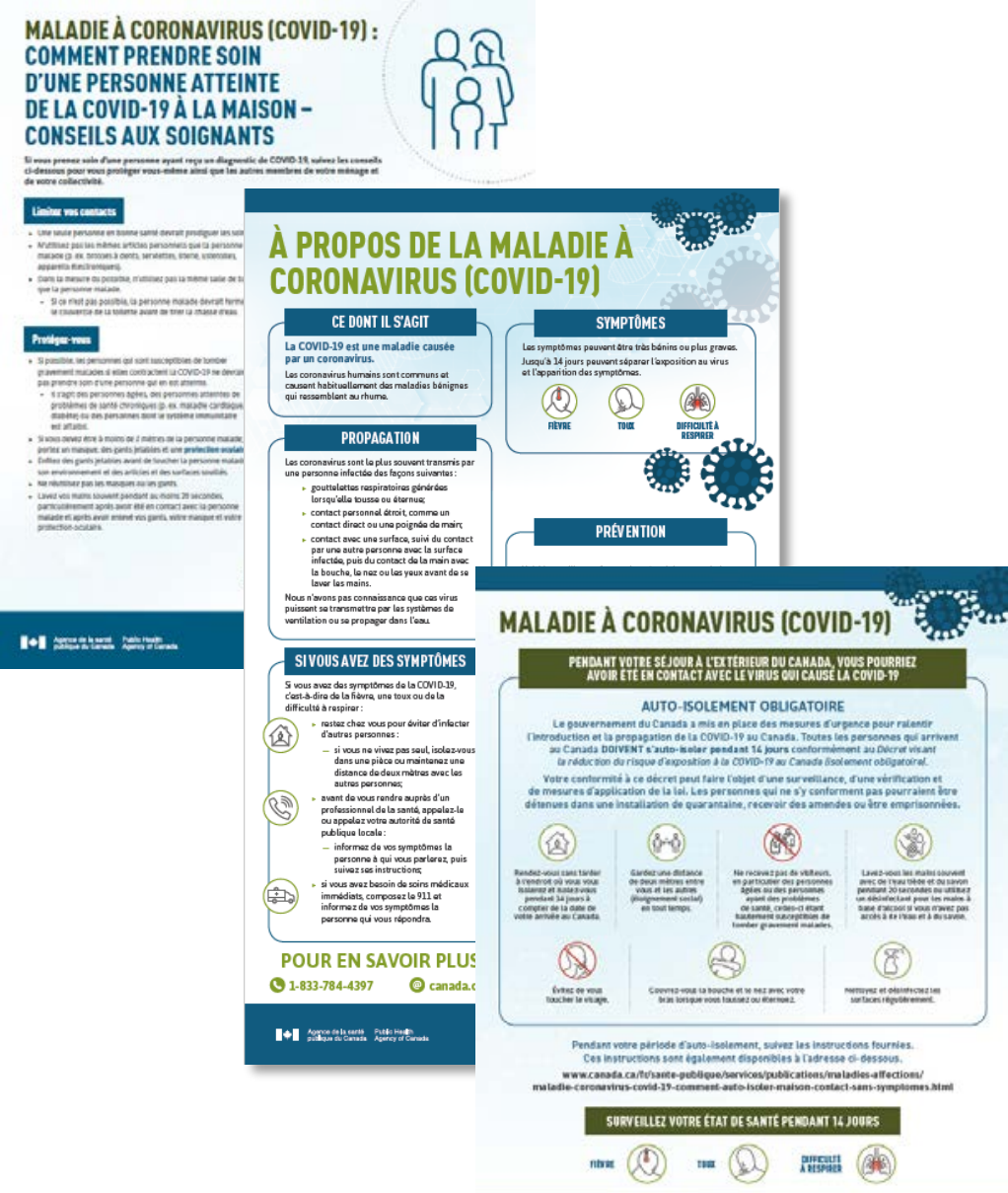

COVID-19

Ressources de sensibilisation

- Obtenez les dernières lignes directrices et ressources de sensibilisation

- Certaines ressources sont disponibles en plusieurs langues

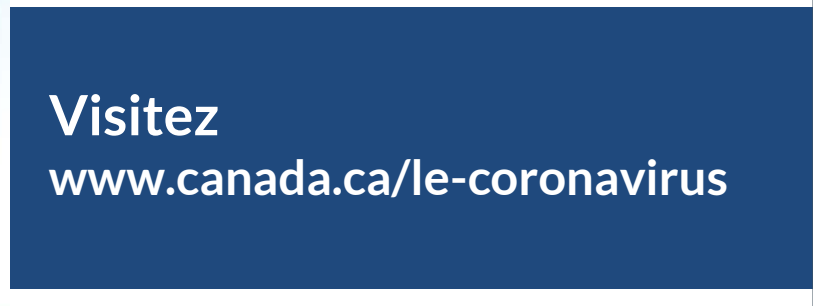

